\title{
Science in the time of coronavirus
}

\author{
Despite the incredible challenges, the science research community is stepping up to address the coronavirus \\ pandemic in multiple ways.
}

U nprecedented. A word that sums up the global COVID-19 pandemic that is currently touching almost all of us. Our hearts go out to those who are sick and suffering, and to those who have lost family members, colleagues and friends.

At the time of writing (mid-March) the number of cases in the United States (where Nature Methods' main office is located) is rapidly growing, and a dark reality is setting in. Stock markets are tanking and the economic impacts will likely be devastating. Pharmacies and supermarkets are emptied of hand sanitizer, paper goods and food. Schools are mostly closed, leaving some parents with no option but to drop work and other families worrying about how they will feed their children. Universities have told students not to come back after spring break while, at the same time, travel restrictions and visa issues have left some stranded. Just about every event anyone has looked forward to is canceled. And the question at the forefront of everyone's mind is: when will it all end; when will normal life resume?

In many ways our modern society is well equipped to cope with the disruption that comes with a pandemic. Many office workers - our editorial team included — already used to flexible working and to connecting with colleagues around the world via video conferencing and messaging, can continue as usual from home without too much interruption. But many scientists whose lives are centered on the laboratory have found their work massively disrupted. Experiments are being put on hold for an indefinite amount of time, setting back untold research projects. Many labs are struggling to maintain basic upkeep of sensitive equipment and animal facilities, and facing hard decisions of what to let go. Technicians worry that they won't be paid, and graduate students worry that they won't graduate. As scientific conferences are canceled, the face-to-face opportunities to share research, make new collaborations and network for jobs are being squelched. And of course almost all of us are having trouble focusing on any kind of work when the news about the pandemic (and fears of falling ill) gets worse with every hour.

At our currently purely virtual office, we are actually seeing an uptick in the number of new submissions to Nature Methods, likely a result of many researchers taking 'social distancing' as an opportunity to focus on writing papers. Some researchers will find themselves with more time than usual to review papers, even welcoming such a distraction, while others will find it completely impossible to take on any such assignments. We sincerely apologize if a request to review a paper seem insensitive during this time - this is not at all our intention. We are trying to maintain a semblance of normalcy and keep up business as usual as long we can sustain it. At the moment it is impossible to predict what the longer-term impacts on research and publication will be.

And yet, all over the world, it is immensely heartening to see how scientists are stepping up to fight this battle and prevent future pandemics. Some labs have taken up the call to donate their supplies or even assist with COVID-19 testing; others are using their skills and knowledge to develop novel diagnostics or to screen for drugs. Labs are openly sharing data that they would normally have fiercely guarded before publication. New collaborations are being rapidly forged between universities, and between researchers from different fields and with different expertise, to study, treat and try to curb the virus (for example: https://news.harvard.edu/ gazette/story/2020/02/harvard-and-chinacollaborate-on-coronavirus-therapies/).

Many basic research labs quickly tuned their priorities to study aspects of SARSCoV-2. For example, recent advances in sequencing technology allowed researchers to rapidly compare viral genomes from patients at the early stages of the outbreak to trace its origin to bats - knowledge that could help prevent the future outbreak of another novel coronavirus. In another example, several structures of the virus's spike glycoprotein (responsible for its binding to host cells) were solved at high resolution by cryoelectron microscopy in just weeks. These results could be instrumental for the development of vaccines and antiviral therapeutics. But it is also a stark reminder that none of this would have been possible without recent technological developments in basic molecular biology and in cryo-EM technology.

Many publishers, including Springer Nature, the publisher of Nature Methods, have committed to a consensus statement on data sharing during public health emergencies. Springer Nature journals are fast tracking the review and publication of COVID-19 research and making all COVID-19 research free to access.

It is unsurprising that there is a high level of potentially disastrous misinformation floating around, ranging from the rumor in France that cocaine cures coronavirus to the claim by some talk show pundits in the US that the pandemic is a hoax. Preprint servers have been inundated with coronavirus manuscripts of wildly varying quality, prompting site managers to display highly visible banners reminding readers that these reports have not been peer-reviewed. Now more than ever, we need to rely on the tried and tested process of peer review to help clinicians, policymakers, journalists and the public quickly assess what is quality work and what is not. We also encourage expert scientists to correct misinformation flying around social media, to answer calls from journalists to help translate technical findings to the general public, and to support policymakers in making sound public health decisions.

If this current crisis has any silver lining, we are hopeful that basic scientific research will emerge stronger, more collaborative, more transparent, better funded and more respected by the public than ever. It has literally become a matter of life and death.

Stay positive, safe and healthy.

Published online: 2 April 2020 https://doi.org/10.1038/s41592-020-0807-y 